Neural modelling

Neural Modelling: Electrical Signal Processing in the Nervous System. By R. J. MacGregor and E. R. Lewis. Pp. 474. (Plenum: New York, 1979.) \$30.

NEURAL MODELling is an activity carried out by theoretical biologists, mathematicians, bioengineers, computer types and some physiologists, the aim of which is to provide insights into how brains work. As such, it has aroused considerable misgivings, to say the least, in the minds of many, if not most, experimental neurobiologists. One distinguished neurophysiologist maintains, perhaps with tongue in cheek, that neurobiology is "totally nonmathematical"; another that mathematical neurobiology is mostly wrong, long on formal theory, but short on facts, and that what is not wrong is trivial. One can perhaps understand, if not sympathise with such feelings, given that most neurobiologists are profoundly ignorant of advanced mathematics, and have formed a rather stereotyped view of the neurotheorist, perhaps not unjustified, as one not really interested in, nor capable of distinguishing 'real' facts.

It is against such a background that this book should be seen. MacGregor and Lewis have tried to cover everything from the biophysical properties of the neuronal membrane to the computational aspects of neocortical nets, in some fifteen chapters comprising 380 pages. Chapters 1 and 2 provide a quick introduction to the neurone doctrine, that the brain is made up of a coupled nerve cells interacting across specialised synapses, and to the notion of the unit compartment: that the critical element in modelling electrical signal processing in cells and in nets is a patch of membrane, rather than an entire cell.

Chapters 3 and 4 cover in some detail the biophysics associated with the generation of transmembrane resting potentials. Readers will find in here a good discussion of the Nernst-Planck and Goldman equations, of Donnan equilibria, of ionic and electrogenic pumps, and the like.

Chapters 5-7 follow this up through the Eccles equivalent circuit for postsynaptic membrane potential changes, the Fuortes-Hodgkin-Baylor models for photoreceptor transduction, Rall's work on cable electrotonus, the Hill-Monnier-Rashevsky theories of suprathreshold excitation, to the Hodgkin-Huxley equations, but not beyond.

There follows a radical change of pace and style, as the authors begin the treatment of neuronal nets. Chapter 8 describes current work on analogue computer simulations of small nets of idealised neurons, called neuromines, and their utilisation in the modelling of invertebrate neuronal structures. Chapters 9 and 10 describe applications of stochastic process theory to model neuronal firing patterns, and the various statistical techniques which have been used to discern regularities in the firing patterns of single and paired cells. Chapters $11-13$ deal with the dynamical properties of largescale nervous activity, seen through Monte Carlo simulations, continuum field theories, or digital computer simulations. Chapter 14, which is very short, discusses briefly, slow potentials and the EEG.

Chapter 15 deals with a number of attempts to understand the functioning of such neuronal structures as the visual neocortex by Pitts and McCulloch, and by Marr; the generation of thalamic and hippocampal rhythms, by Anderson et at.; and the brain-stem reticular formation; and there is a brief mention of lateral inhibition in the retina. Finally, chapter 16 closes with a few methodological comments about modelling in general, and brain modelling in particular.

In my opinion, the authors have failed to provide a sufficiently penetrating account of the field of neuronal

\section{Chromatographic methods}

\section{Laboratory Handbook of Chromatographic and Allied Methods.} Edited by O. Mikeš. Pp. 764. (Ellis Horwood/Wiley: Chichester, UK, 1979.) $£ 38.50$.

THIS is an excellent addition to the numerous practically oriented books in the Ellis Horwood Series in Analytical Chemistry. Dr Mikeš has enlisted the help of 13 of his colleagues, all employed in the Czechoslovak Academy of Sciences, to produce a completely rewritten version of his Laboratory Handbook of Chromatographic Methods (1966). I consider the present book to be a worthy successor to E. and M. Lederer's Chromatography $(1953,1957)$.

The "allied methods" alluded to in the title are countercurrent distribution and electromigration, and one chapter is allocated to each. The chapter on countercurrent distribution is clear about matters of principle. However, it ignores the dramatic usefulness of the technique in determining the structure of the antibiotic peptides and in the purification of transfer RNAs by Holley and colleagues. Moreover, no mention is made of Alderweireldt's approach, which culminated in the development of 'steadystate' machines; the example given of use of such a machine is undocumented and fails completely to illustrate its capabilities modelling, up to mid-1977. It is, of course, always a problem in seeking to convey the nature of a discipline, to choose between breadth or depth. To my taste, only chapters 3 and 4 cover the material in sufficient depth as to be interesting. The others are so superficial as to be relatively boring for anyone who knows anything about the subject matter. In addition to this, I feel that the book is written from the wrong perspective, from the bottom up as it were, instead of from the top down. Over the past 5 years or so, it has become apparent to many neurotheorists, that trying to deduce what is going on in neuronal circuits from the properties of neurones and synapses is not very profitable, and that one has to understand in some detail the computational problems which have to be solved by the system and the algorithms used, before one can get to questions of how such algorithms are embodied in neuronal circuits. This is only hinted at in the later chapters of this book. A really good book on the subject of neuronal modelling remains to be written.

Jack Cowan

Jack Cowan is Professor of Theoretical Biology at the University of Chicago, Illinois.

for finding needles in haystacks. The chapter covering electrophoresis is unclear on matters of principle and inadequate in coverage.

By contrast, the 10 chapters relating to chromatography proper provide excellent coverage both of principle and of manipulative practice. Analytical applications described in the text are by way of example only, but the references to reviews and original papers make it easy to find out how any particular technique has been used to deal with particular classes of organic or inorganic substances. There are some notable omissions. Liquid-liquid chromatography on columns and preliminary concentration from crude mixtures by displacement are scarcely mentioned. Although carrier displacement is discussed in principle, no mention is made of Sanger's brilliant application of it to oligonucleotides. Indeed, there is little practical advice in the whole work about detecting or measuring radioactive substances in chromatography. However, in general, the treatment of chromatography is excellent and the practical detail and careful and extensive bibliography will make this book a good investment for the library of every establishment where chemical analyses are carried out, and particularly for those establishments where versatility is demanded.

R.L.M. Synge

R.L.M. Synge is Honorary Professor of Biology at the University of East Anglia, Norwich, UK. 